\title{
Traumatic Lumbar Puncture is Unlikely to Reduce Modern Molecular Detection of HSV Encephalitis (HSVE)
}

Kacy A Ramirez ${ }^{1,2,3,4^{*}}$, Rebecca Thompson ${ }^{2}$, Ishita Pareek ${ }^{2}$, Carson House ${ }^{2}$, Noel T. Lenny ${ }^{2,4}$, Anami Patel ${ }^{1,2,4}$ and John P DeVincenzo ${ }^{1,2,4,5}$

${ }^{1}$ Department of Pediatrics, University of Tennessee Health Science Center, Memphis, TN, United States

${ }^{2}$ Methodist Le Bonheur Children's Hospital, Memphis, TN, United States

${ }^{3}$ Department of Infectious Disease, St. Jude Children's Research Hospital, Memphis, TN, United States

${ }^{4}$ Children's Foundation Research Institute, Memphis, TN, United States

${ }^{5}$ Department of Microbiology, Immunology and Biochemistry, University of Tennessee Health Science Center, Memphis, TN, United States

*Corresponding author: Kacy A Ramirez, MD, Pediatric Infectious Disease, Department of Pediatrics, Wake Forest Baptist Medical Center, Medical Center Boulevard, Mead's Hall, Winston-Salem NC 27157, USA, Tel: 336-716-5261; Fax: 336-716-9699; E-mail: kramirez@wakehealth.edu

Received date: March 16, 2018; Accepted date: March 29, 2018; Published date: April 5, 2018

Copyright: ( $) 2018$ Ramirez KA, et al. This is an open-access article distributed under the terms of the Creative Commons Attribution License, which permits unrestricted use, distribution, and reproduction in any medium, provided the original author and source are credited.

\begin{abstract}
Polymerase chain reaction (PCR) is the gold-standard for diagnosing HSVE, but may be inhibited by blood contamination. We mimicked traumatic lumbar puncture and measured its effect on the molecular detection of HSV-1 in CSF. Clinically meaningful reductions in sensitivity were not observed. Pre-PCR processing allows sensitive detection of HSVE despite traumatic lumbar puncture.
\end{abstract}

Keywords: Traumatic lumbar puncture; Traumatic tap; HSV; HSV encephalitis; PCR inhibition

\section{Introduction}

The detection of HSV DNA in the cerebrospinal fluid (CSF) by PCR is the gold standard for diagnosing HSVE [1,2], which can produce very small quantities of DNA. Therefore, PCR detection must be extremely sensitive. Overall sensitivities and specificities of CSF PCR in neonatal HSV disease have ranged from 71-100\% [1]. Even higher sensitivities and specificities (98 and $94 \%$, respectively) of the assay have been reported in adults with HSVE when compared to brain biopsy [2], and sensitivity of the assay is maintained up to a week after initiating antiviral therapy $[2,3]$.

The sensitivity of real-time PCR is affected by the quality of target nucleic acids, reaction conditions, and the presence of extraneous matter [4]. Amplification can be inhibited by contaminants present in blood, including haemoglobin, lactoferrin, and immunoglobulin's [5,6]. Taq and AmpliTaq Gold DNA polymerases (utilized widely in Hot Start/Fast Start "master mix" real-time PCR protocols) are inhibited by as little as $14,000 \mathrm{RBC} / \mathrm{mm}^{3}$ [6]. Heme, particularly the degenerated heme complex, is considered a universal PCR inhibitor. Release of iron ions from heme contaminants affects ion balance and disturbance of DNA polymerase activity and primer/probe annealing [6]. Hemin is a competitive inhibitor of target DNA, and immunoglobulin $\mathrm{G}$ may bind directly to ssDNA, further inhibiting nucleic acid amplification [5].

Modern real-time PCR molecular diagnostic assays typically attempt to avoid these inhibitory effects by processing techniques applied prior to amplification, detection and quantification of the PCR product [4]. Specimen processing techniques include cell separation and removal of visible RBC's from CSF by centrifugation. Extraction techniques concentrate nucleic acids and remove/neutralize PCR inhibitors. Column-based solid-phase (DNA manual mini-kits) or automated (QIAGEN EZ-1 or NucliSENS EasyMAG) magnetic resin/ silica DNA extraction and purification methods are effective and commonly employed [6]. Furthermore, PCR chemistry has been optimized through utilization of specific buffers, $\mathrm{pH}$, salt conditions, and amplification facilitators (bovine serum albumin is used to bind heme, DMSO is used to destabilize DNA) [6].

Traumatic lumbar punctures occur frequently, approaching 14-40\% [7-9]. The visible threshold of detection (CSF visibly pink tinged) is defined as $>400 \mathrm{RBC} / \mathrm{mm}^{3}$. Published ranges of blood within the CSF after traumatic LPs include 1,000-23,000 RBCs $/ \mathrm{mm}^{3}$ (median 3,000-4,000 RBCs $/ \mathrm{mm}^{3}$ ) [9]. Viral-induced haemorrhage into the CSF contributes to this contamination [10].

Newer molecular diagnostic techniques including pre-PCR processing may reduce PCR inhibition by blood products. Within the CSF, the clinically applicable limitations of sensitivity by blood products on real-time PCR have not been evaluated. We mimicked traumatic lumber puncture and studied the effects of various clinicallyrelevant concentrations of human blood on the quantitative real-time PCR detection of HSV-1 in human CSF.

\section{Materials and Methods}

Human CSF was obtained and pooled from patients undergoing routine ventriculoperitoneal shunt placement. It was determined free of RBC's and detectable HSV virus within the clinical laboratory by automated $\mathrm{CBC}$ technology and real-time PCR.

\section{Sample treatment techniques prior to Real-time PCR}

Normal pooled human CSF was spiked with clinically-relevant HSV-1 concentrations (Figure 1) derived from human fetal lung fibroblast (MRC-5) tissue culture. For experimental condition A (ECA), whole blood collected in EDTA and serially diluted in blood buffer (CELL-DYN Sapphire, Germany) was then added to this spiked CSF to produce final concentrations of 200,000 to $0.2 \mathrm{RBC} / \mathrm{mm}^{3}$ (Figure 
insert $^{*}$ ). For experimental condition B (EC-B), samples were prepared as EC-A but followed by $30 \mathrm{~s}$ of mini-centrifugation and removal of supernatant. For experimental condition C (EC-C), whole blood was haemolysed (frozen on dry ice for $10 \mathrm{~min}$ followed by $30 \mathrm{~s}$ of vortexing and repeated three times). This haemolysed blood was then added to spike CSF followed by $30 \mathrm{~s}$ of mini-centrifugation for each prepared specimen (similar to EC-B). A positive control included pooled human CSF spiked with HSV-1 and RBC diluent blood buffer. Negative controls included: (1) pooled human CSF, and (2) RBC diluent blood buffer.

\section{HSV-1 detected by PCR in patients with HSVE (2004-2013)}

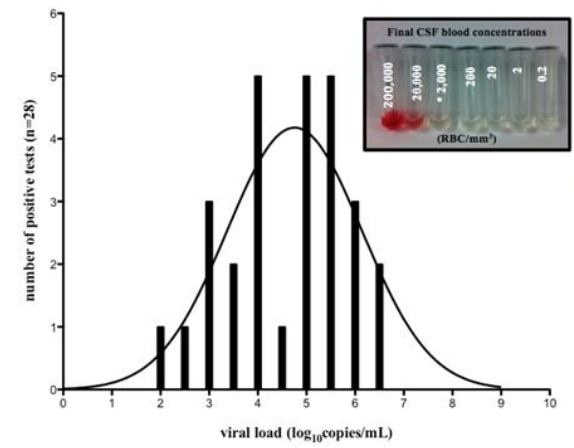

Figure 1: The solid bars represent viral load data from patients with HSV-1 induced encephalitis diagnosed from 2004-2013. The solid line is the computed normalized distribution of the viral loads. The maximum lowering of measured viral load $(0.53 \log 10$ copies $/ \mathrm{mL})$ contributed experimentally by $2,000 \mathrm{RBC} / \mathrm{mm}^{3}$ (inset, ${ }^{*}$ ) could potentially render a false negative test in only $1-2$ of $28(3.6-7.1 \%)$ of our patient population with HSVE. ${ }^{*}$ Inset: pooled CSF containing various experimentally-added concentrations of non-haemolysed whole blood $\left(\mathrm{RBC} / \mathrm{mm}^{3}\right)$.

HSV-1 viral DNA was extracted from $200 \mu \mathrm{L}$ of these processed samples utilizing the automated Biorobot EZ-1 instrument/Virus Mini Kit v2.0 (QIAGEN, Germantown, Maryland, USA) and eluted into 60 $\mu \mathrm{L}$ of sterile water. The concentration within the CSF of HSV-1 spiked into samples was chosen to mimic the mean viral load in clinical patients with HSV-1 encephalitis, generated by the same real-time PCR utilized for this experimental study.

\section{Detection and quantification of HSV-1 DNA by Real-time PCR}

Detection of extracted HSV-1 DNA pol conserved gene segment was carried out by the Light Cycler 2.0 (Roche, Indianapolis, IN, USA) via real-time PCR utilizing a home-brew assay based on hybridization probe technology. As previously described [11], forward and reverse primer HSV polymerases were used to amplify a 140 base-pair product. Real-time detection and melting temperature differentiation of HSV-1 and 2 was determined with a pair of fluorescence-labelled probes. The reaction mixture was prepared in a controlled access reagent preparation room and consisted of $15 \mu \mathrm{L}$ of PCR master mix and $5 \mu \mathrm{L}$ of DNA extract per CSF sample. Cycling conditions and melting curve analysis after amplification were then determined [11]. Samples were run in duplicate with a negative and positive control, and were considered positive if both replicates had Ct values $<40$ and negative if there was no amplification within 40 amplification cycles. Quantification was based on external standard curves made from highly-concentrated, purified and deactivated whole HSV virus isolated from cell culture and quantified via pico green (Zeptometrix, Buffalo, NY, USA).

\section{Statistical methods}

After testing for normality of distribution, the Wilcoxon Rank Sum (Mann-Whitney U) test compared median viral load $(\log 10$ copies $/ \mathrm{mL}$ ) between experimental groups and control (CSF + HSV, no blood). Two-tailed $\mathrm{p}$ values $<0.05$ were considered statistically significant.

\section{Human subjects protection}

IRB approval was obtained for all studies as appropriate.

\section{Results}

Despite the addition of widely differing concentrations of haemolysed and non-haemolysed blood and the application of different processing techniques (the absence of processing v/s sample centrifugation prior to extraction), no blood concentration significantly affected PCR detection of HSV-1 DNA in CSF compared to control (CSF+HSV without blood) [Mean $4.60 \log 10$ copies/mL (CI: 4.04-5.17)], p all $>0.05]$.

A trend toward reduction in the quantitative detection of HSV-1 occurred in the HSV spiked CSF containing 2,000 $\mathrm{RBC} / \mathrm{mm}^{3}$ of haemolysed blood (EC-C) (Figure insert ${ }^{*}$ ). However, this did not reach statistical significance [maximum lowering of measured viral load was $0.53 \log 10$ copies/mL of HSV DNA, ( $\mathrm{p}$ all $>0.05)]$. When applied to a calculated normalized distribution of the clinical mean CSF viral load from our 28 patients with HSVE quantitatively detected by real-time PCR in our laboratory from 2004-2013 (Figure 1), this maximum lowering of measured viral load could potentially render a false negative test in only 1-2 of 28 (3.6-7.1\%) patients with HSVE.

\section{Discussion}

Traumatic LP occurs often $[8,9]$, and haemorrhagic CSF, secondary to viral-induced necrosis in patients with HSVE, is well described [10]. Most real-time PCR assays, including ours, utilize commercially available TaqDNA polymerases, which are sensitive to heme-induced inhibition [4-6]. Thus, blood contamination interferes with PCR amplification. Whether this phenomenon occurs to a clinically meaningful extent using modern pre-PCR processing techniques and PCR assays has not been evaluated. We demonstrated that despite the phenomenon of PCR inhibition by blood contaminants cited by manufacturers, modern molecular diagnostic pre-PCR processing techniques, most importantly improved extraction capabilities and optimal PCR chemistry, likely overcome the previously-observed PCR inhibition by blood products. This allows the sensitive detection of HSVE despite traumatic lumbar puncture.

In an effort to optimize sensitivity of molecular diagnostic assays, sample-processing techniques of visibly bloody CSF specimens, including centrifugation prior to extraction, are widely practiced. But some degree of limitation of generalizability of these results warrants discussion. Performance of PCR varies amongst laboratories given the lack of inter-laboratory quantitative and non-quantitative standards and the use of single-lab ("home-brew") developed assays [6]. Reagent 
buffers and amplification facilitators utilized to provide optimal PCR chemistry may also vary. However, results of our study should not undermine the importance of modern extraction procedures, in addition to PCR chemistry, employed to reduce such theoretical inhibition.

Modern extraction techniques are widely utilized [6] and are equally effective [12]. Manual column-based solid-phase mini-kits or the automated biorobots, which use magnetic resin/silica for DNA extraction and purification, have replaced older Chelex 100 resin or organic phenol chloroform extraction techniques, providing concentrated and more highly purified DNA given their ability to more effectively adsorb water soluble complexes of ferric heme and serum proteins [6]. Modern pre-PCR processing techniques may not be standardized, but optimal chemistry and extraction is generally utilized in commercial kits and assays, allowing sensitive detection of HSV in CSF despite the presence of blood products.

The morbidity and mortality of HSVE remains unacceptably high. Up to $69 \%$ of neonates and $44-62 \%$ of children and adults will suffer long-term neurological impairment despite treatment (acyclovir) $[10,13]$. Early initiation of acyclovir and treatment until the cerebrospinal fluid viral load becomes undetectable is prudent as rapid reduction and suppression of viral replication is necessary to improve clinical outcomes in patients with HSVE $[1,14]$. Quantitative real-time polymerase chain reaction (PCR) molecular techniques enable earlier detection and initiation of therapy for HSVE before widespread dissemination of virus and allow the clinician to potentially monitor response to treatment. Our study has demonstrated that despite traumatic lumbar puncture, the essential sensitivity of PCR in CSF is maintained in patients with HSVE.

\section{Conclusions}

Advanced pre-PCR processing techniques including sample treatment (extraction) and optimal PCR chemistry appear to overcome the previously observed PCR inhibition by blood products allowing sensitive detection of HSVE despite traumatic lumbar puncture.

\section{Acknowledgement}

We would like to thank our research assistant Lisa Harrison (University of Tennessee, Memphis TN) for her contribution to the molecular diagnostic methods of this project.

\section{References}

1. Kimberlin DW, Lakeman FD, Arvin AM, Prober CG, Corey L, et al (1996) Application of the polymerase chain reaction to the diagnosis and management of neonatal herpes simplex virus disease. J Infect Dis 174: 1162-1167.

2. Lakeman FD, Whitley RJ (1995) Diagnosis of herpes simplex encephalitis: application of polymerase chain reaction to cerebrospinal fluid from brain-biopsied patients and correlation with disease. J Infect Dis 171: 857-863.

3. Ziyaeyan M, Alborzi A, Borhani Haghighi A, Jamalidoust M, Moeini M, et al. (2011) Diagnosis and quantitative detection of HSV DNA in samples from patients with suspected herpes simplex encephalitis. Braz J Infect Dis 15: 211-214.

4. Radstrom P, Lofstrom C, Lovenklev M, Knutsson R, Wolffs P (2008) Strategies for overcoming PCR inhibition. CSH Protoc: pdb top20.

5. Al-Soud WA, Radstrom P (2001) Purification and characterization of PCR-inhibitory components in blood cells. J Clin Microbiol 39: 485-493.

6. Hedman J, Radstrom P (2013) Overcoming inhibition in real-time diagnostic PCR. Methods Mol Biol 943: 17-48.

7. Lyons TW, Cruz AT, Freedman SB, Neuman MI, Balamuth F, et al. (2017) Interpretation of Cerebrospinal Fluid White Blood Cell Counts in Young Infants With a Traumatic Lumbar Puncture. Ann Emerg Med 69: 622-631.

8. Pappano D (2010) "Traumatic tap" proportion in pediatric lumbar puncture. Pediatr Emerg Care 26: 487-489.

9. Shah KH, Richard KM, Nicholas S, Edlow JA (2003) Incidence of traumatic lumbar puncture. Acad Emerg Med 10: 151-154.

10. Whitley RJ (2006) Herpes simplex encephalitis: adolescents and adults. Antiviral Res 71: 141-148.

11. Burrows J, Nitsche A, Bayly B, Walker E, Higgins G, et al. (2002) Detection and subtyping of Herpes simplex virus in clinical samples by LightCycler PCR, enzyme immunoassay and cell culture. BMC Microbiol 2: 12 .

12. Dundas N, Leos NK, Mitui M, Revell P, Rogers BB (2008) Comparison of automated nucleic acid extraction methods with manual extraction. J Mol Diagn 10: 311-316.

13. Kimberlin DW, Lin CY, Jacobs RF, Powell DA, Corey L, et al. (2001) Safety and efficacy of high-dose intravenous acyclovir in the management of neonatal herpes simplex virus infections. Pediatrics 108: 230-238.

14. Mejias A, Bustos R, Ardura MI, Ramirez C, Sanchez PJ (2009) Persistence of herpes simplex virus DNA in cerebrospinal fluid of neonates with herpes simplex virus encephalitis. J Perinatol 29: 290-296. 